Gut and Liver, Vol. 12, No. 4, July 2018, pp. 385-392

\title{
Optical Diagnosis for Colorectal Polyps: A Useful Technique Now or in the Future?
}

Ignasi Puig ${ }^{1}$ and Tonya Kaltenbach ${ }^{2}$

${ }^{1}$ Division of Gastroenterology, Althaia, Xarxa Assistencial Universitària de Manresa, Universitat Internacional de Catalunya, Barcelona, Spain, ${ }^{2}$ Division of Gastroenterology, Department of Medicine, VA San Francisco and the University of California San Francisco, San Francisco, CA, USA

In the last few years, interest in the optical diagnosis of colorectal polyps has increased among gastroenterologists. Several studies have shown that the optical diagnosis of small colorectal polyps is safe and feasible in routine clinical practice and is comparable to histopathology. The Narrowband Imaging International Colorectal Endoscopic Classification provides a validated criterion for the classification of neoplastic and nonneoplastic polyps as well as polyps with deep submucosal invasion using narrow band imaging during realtime colonoscopy. The aim of the present review is to assess the current evidence for and limitations of optical diagnosis and to propose a systematic approach for transferring research findings to patient care. (Gut Liver 2018;12:385392)

Key Words: Colonoscopy; Colonic polyps; Optical imaging

\section{INTRODUCTION}

Gastroenterologists are currently debating the possibility of a paradigm shift in the colonoscopic management of diminutive colorectal polyps. The replacement of routine postpolypectomy pathological analysis of diminutive polyps with real time endoscopic optical diagnosis, also known as the predict-resectand-discard strategy, is an attractive, cost-effective approach to colorectal cancer screening which reduces the time associated with post-polypectomy specimen retrieval and the cost of histopathology. ${ }^{1,2}$

A number of studies, including several meta-analyses, ${ }^{3,4}$ have shown that optical diagnosis of small colorectal polyps is safe and feasible in routine clinical practice and comparable to the current reference standard, histopathology. Experienced endoscopists have achieved 93\% concordance of surveillance intervals made by real-time optical diagnosis and pathology, and a >90\% negative predictive value for rectosigmoid polyps. As the risk of malignancy increases with polyps greater than $1 \mathrm{~cm}$ in size, most studies have evaluated the accuracy of optical diagnosis in polyps $<10 \mathrm{~mm}$. Furthermore, the American Society for Gastrointestinal Endoscopy (ASGE) Technology Committee, in its Preservation and Incorporation of Valuable endoscopic Innovations (PIVI) statement, concentrated exclusively on diminutive polyps ( $\leq 5 \mathrm{~mm}$ ), in order to reduce the risk even further. ${ }^{5}$

The Narrow-band imaging Imaging International Colorectal Endoscopic (NICE) Classification provides a validated criterion for the classification of neoplastic and nonneoplastic polyps as well as those with deep submucosal invasion, using narrow band imaging during real-time colonoscopy (Fig. 1). ${ }^{6,7}$ Other classification systems for narrow-band imaging (NBI) and other narrow spectrum technologies, as well as chromoendoscopy, have also been described for magnified colonoscopy.

Although the resect and discard strategy is still not widely used, endoscopists tend to leave in situ diminutive polyps in the rectum and sigmoid with typical hyperplastic features. In addition, the number of endoscopists who describe optical diagnosis in their colonoscopy reports is increasing, as it may also be useful for unretrieved polyps in which pathological diagnosis is not available. However, training is a prerequisite and certain concepts should be taken into account before optical diagnosis can be used in clinical practice. The aim of the present review is to assess the current evidence and limitations of optical diagnosis, and to propose a systematic approach for transferring the research findings to patient care.

Correspondence to: Tonya Kaltenbach

Division of Gastroenterology, Department of Medicine, VA San Francisco and the University of California San Francisco, 4150 Clement Street (VA111B), Bldg 203, 2A-67, San Francisco, CA 94121, USA

Received on March 22, 2017. Revised on June 5, 2017. Accepted on June 10, 2017. Published online December 28, 2017

pISSN 1976-2283 eISSN 2005-1212 https://doi.org/10.5009/gnl17137

@. This is an Open Access article distributed under the terms of the Creative Commons Attribution Non-Commercial License (http://creativecommons.org/licenses/by-nc/4.0) which permits unrestricted non-commercial use, distribution, and reproduction in any medium, provided the original work is properly cited. 


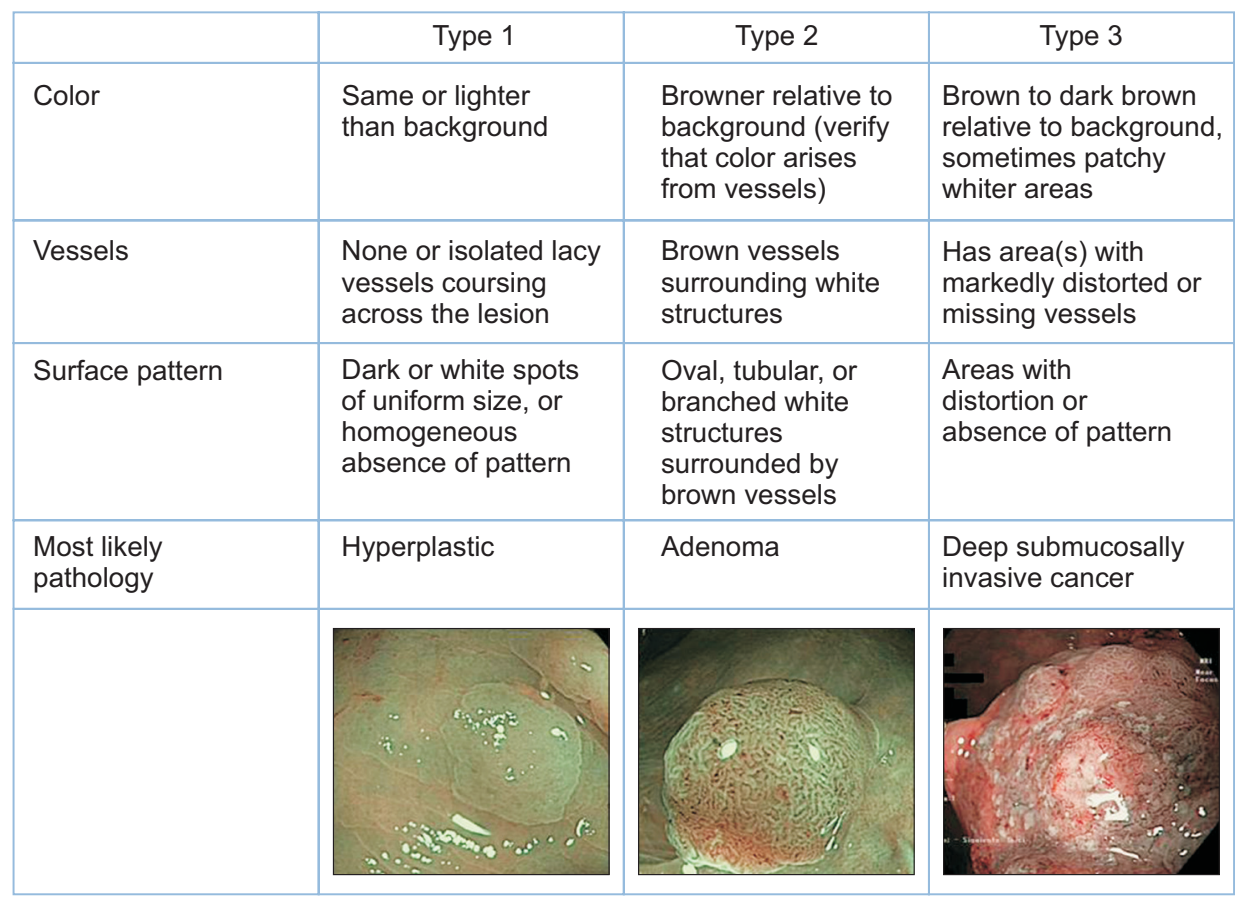

Fig. 1. Narrow-band imaging International Colorectal Endoscopic (NICE) classification. Adapted from Hayashi N, et al. Gastrointest Endosc 2013;78:625-632. ${ }^{7}$

\section{ARE WE READY TO IMPLEMENT OPTICAL DIAGNOSIS IN ROUTINE CLINICAL PRACTICE?}

Several studies from both Eastern and Western countries, and either with or without magnification, have demonstrated a high diagnostic accuracy for distinguishing neoplastic from nonneoplastic lesions. A systematic review and meta-analysis including 28 studies that used NBI for optical diagnosis of colon polyp histology in real-time found a high diagnostic accuracy (sensitivity, 91\%; specificity, 83\%; hierarchical summary receiver operating characteristic curve, 0.92). ${ }^{3}$ In addition, a recent meta-analysis confirmed that the thresholds established by the ASGE PIVI for real-time endoscopic assessment of the histology of diminutive polyps had been met, at least with NBI optical biopsy, by endoscopists who were expert in using this advanced imaging technology and when assessments were made with high confidence. ${ }^{4}$

The NICE classification has shown to be useful in assessing the most clinically relevant approaches: leave hyperplastic diminutive polyps of the rectal and sigmoid colon, remove adenomas/superficial adenocarcinomas and proximal hyperplastic polyps, and biopsy and refer to surgery lesions with deep submucosal invasion (Fig. 1). This classification has been widely accepted in both Western and Eastern countries and has been reproduced in other studies. ${ }^{8-10}$ The NICE classification has some limitations: it does not attempt to distinguish villous elements or the grade of dysplasia within adenomas, or to differentiate sessile serrated adenoma/polyps (SSA/P) from hyperplastic polyps. However, in polyps $<5 \mathrm{~mm}$, the presence of villous and/ or high-grade dysplasia is rare (around $0.2 \%$ to $5 \%$ ) $^{11,12}$ as is the presence of SSA/P (around 0.7\%). ${ }^{13}$

\section{OPTICAL DIAGNOSIS OF SERRATED POLYPS}

Clinical interest in serrated polyps has increased in recent years, as up to $30 \%$ of all colorectal cancers have been shown to arise from these polyps. ${ }^{14} \mathrm{SSA} / \mathrm{P}$, traditional serrated adenomas and hyperplastic polyps are included in the serrated lesion class. Diagnosis of SSA/P is based on $\geq 1$ unequivocal distorted, dilated glands and/or horizontally branched crypt. ${ }^{15}$ However, agreement on diagnosis among pathologists has been shown to be suboptimal ${ }^{16,17}$ and therefore distinguishing SSA/P from hyperplastic polyps endoscopically is challenging because of the variations in pathologic diagnoses.

A recent study incorporated SSA/P features into the NICE classification. ${ }^{18}$ Without magnification, the NICE criteria were combined with four serrated features (clouded surface, indistinctive borders, irregular shape and dark spots inside crypts). If a NICE 1 or 2 polyp had at least two serrated features, it was considered a SSA/P (Fig. 2). The validation study used still image review and showed that, in polyps $<5 \mathrm{~mm}$ with a high confidence assessment, the accuracy for distinguishing SSA/P from non SSA/P and the NPV for neoplasia (including adenoma and SSA/P) both reached 91\%.

As mentioned above, the presence of SSA/P in lesions $<5 \mathrm{~mm}$ is very low. According to a prospective study in Japan, ${ }^{13}$ the proportion of SSA/P increases with size $(\leq 5 \mathrm{~mm}, 0.7 \% ; 6$ to 9 $\mathrm{mm}, 29 \%$; $\geq 10 \mathrm{~mm}, 70 \%)$. However, until these endoscopic and pathologic distinctions are further described, investigated and reproduced, it may be necessary to remove all lesions with SSA/ 
P features and send them for pathological analysis.

\section{HOW CAN OPTICAL DIAGNOSIS BE INTRODUCED INTO CLINICAL PRACTICE?}

The introduction of a systematic approach in order to transfer the research findings into patient care is the next important step. ${ }^{19}$ The suggested measures for implementing the optical diagnosis of diminutive colorectal polyps in clinical practice include:

\section{Use of standardized outcomes for optical diagnosis: the PIVI statement}

The ASGE's PIVI working group established a priori diagnostic thresholds for real-time endoscopic assessment of the histology of diminutive colorectal polyps. ${ }^{5}$ These thresholds are intended to define clinically important roles for imaging technology and acceptable levels of performance; once met, the ASGE supports their use as an alternative paradigm for the management of diminutive polyps in clinical practice.

The two proposed thresholds for optical diagnosis are summarized in Table 1 . The ASGE PIVI statement recommends: ${ }^{5}$

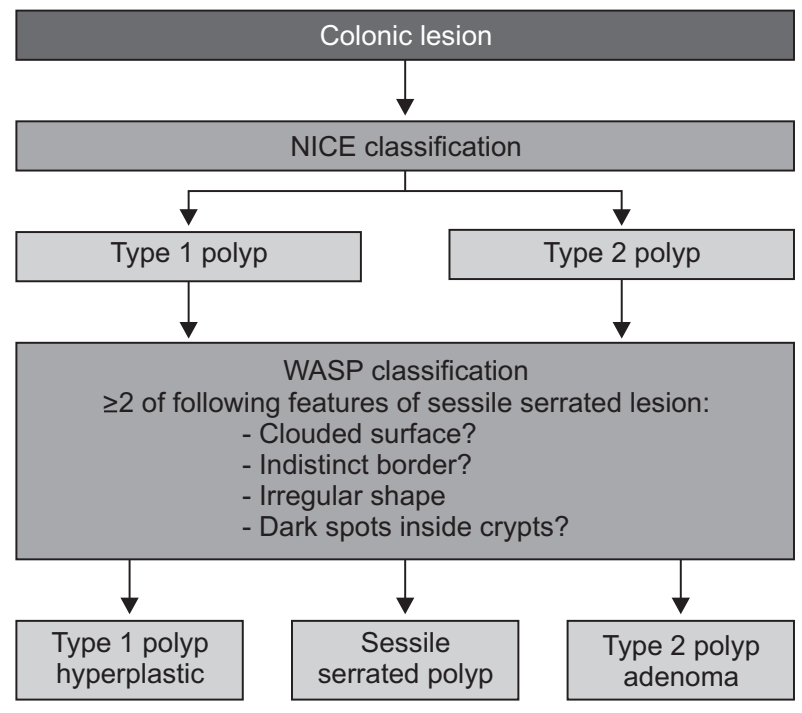

Fig. 2. Workgroup Serrated Polyps and Polyposis (WASP) classification for the optical diagnosis of hyperplastic polyps, sessile serrated lesions and adenomas based on the Narrow-band Imaging International Colorectal Endoscopic classification and four sessile serrated lesion-like features. Adapted from IJspeert JE, et al. Gut 2016;65:963970, with permission from BMJ Publishing Group Ltd. ${ }^{18}$
"In order for colorectal polyps $\leq 5 \mathrm{~mm}$ in size to be resected and discarded without pathologic assessment, endoscopic technology (when used with high confidence) and histopathologic assessment of polyps $\leq 5 \mathrm{~mm}$ in size should provide a $\geq 90 \%$ agreement in the assignment of post-polypectomy surveillance intervals when compared to decisions based on pathology assessment of all identified polyps.

In order for a technology to be used to guide the decision to leave suspected rectosigmoid hyperplastic polyps $\leq 5 \mathrm{~mm}$ in size in place (without resection), the technology should provide a $\geq 90 \%$ negative predictive value (when used with high confidence) for adenomatous histology."

The rationale for optical diagnosis is that diminutive polyps rarely harbor advanced histology, ${ }^{11-13}$ and that pathologists achieve an accuracy of 85\% to 95\% in polyp histology characterization. $^{17,20}$

\section{Level of confidence should be used}

The endoscopist's level of confidence is a crucial factor in optical diagnosis. Only lesions in which the endoscopist has a high level of confidence should be considered for optical diagnosis. The level of confidence in optical diagnosis is key for the success of the strategy in clinical practice. The use of confidence levels allows calibration and standardization between endoscopists with varying levels of diagnostic ability (Fig. 3). Thus, if a polyp lacks clear endoscopic features which preclude confident endoscopic assignment of histology, the endoscopist can still resect and submit it for pathologic assessment.

Various criteria have been proposed for defining the confidence level. "High confidence" can be assigned to optical diagnosis when a polyp has endoscopic color, surface and/or vessel features associated with a specific type of histology in the NICE classification, and no features associated with another type. ${ }^{21}$ High certainty of diagnosis $(\geq 90 \%)$ has also been proposed. ${ }^{6}$ Recently, a prospective study found an association between the time spent determining the optical diagnosis and diagnostic accuracy (Table 2), ${ }^{22}$ and proposed the " 5 -second rule" as a highconfidence criterion.

The addition of Image-Enhanced Endoscopy can also help to evaluate the polyps with high confidence. Both near focus and high magnification have proved useful for increasing the number of lesions that can be assessed with high confidence, although the diagnostic accuracy of both near focus and standard focus reached the PIVI requirements.

Table 1. ASGE PIVI Statement Guidelines: Diagnostic Thresholds for Real-Time Endoscopic Assessment of the Histology of Diminutive Colorectal Polyps

\begin{tabular}{lll}
\hline 1. Resect \& discard & $\leq 5 \mathrm{~mm}$ polyps & $\begin{array}{l}\geq 90 \% \text { agreement in assignment of postpolypectomy surveillance intervals, } \\
\text { using pathology standard }\end{array}$ \\
2. Do not resect & Rectosigmoid hyperplastic $\leq 5 \mathrm{~mm}$ & $\geq 90 \%$ negative predictive value for adenoma
\end{tabular}



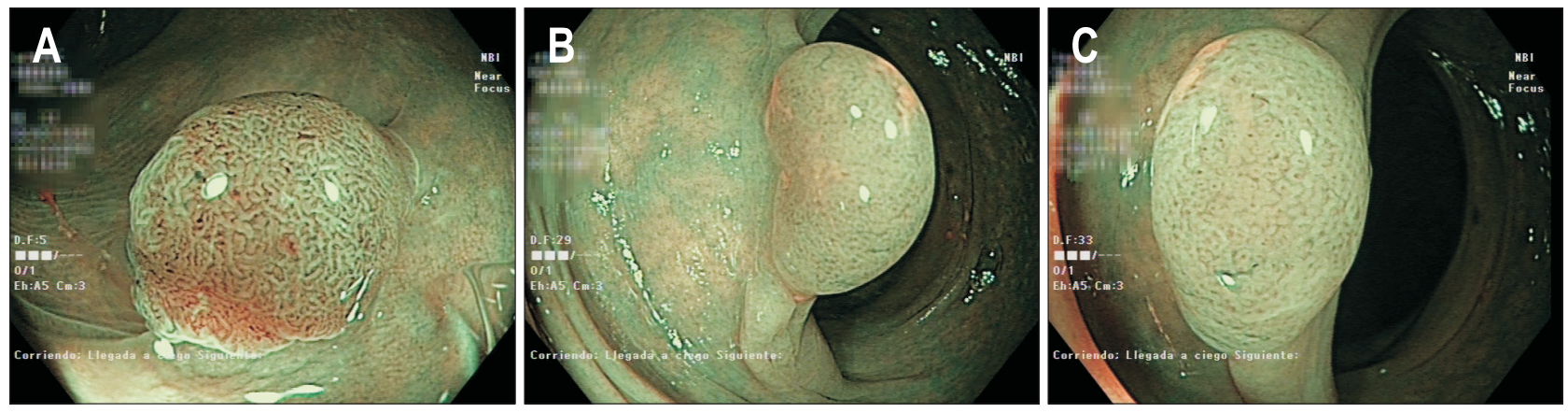

Fig. 3. (A) Sessile (Is) polyp in a rectum. Both an experienced and a novice endoscopist would evaluate it as an adenoma with high confidence. (B, C) Sessile (Is) polyp in a sigmoid colon. An experienced endoscopist would evaluate the image as hyperplastic with high confidence. A novice endoscopist may assess it with low confidence, but if the histology is checked, the learning process continues and the number of high confidence assessments may increase in the future.

Table 2. Five Seconds Rule

\begin{tabular}{ccc}
\hline $\begin{array}{c}\text { Optical diagnosis } \\
\text { time }(\mathrm{sec})\end{array}$ & Accuracy $(\%)$ & $\begin{array}{c}\text { High-confidence } \\
\text { determinations }(\%)\end{array}$ \\
\hline$<5$ & $>90$ & 90 \\
$6-60$ & 85 & 77 \\
$>60$ & 68 & 64 \\
\hline
\end{tabular}

Diagnostic accuracy for distinguishing neoplastic from nonneoplastic lesions and percentage of high-confidence lesions according to the time taken to determine the optical diagnosis.

Adapted from Gupta N, et al. Gastrointest Endosc 2013;77(5 Suppl): AB553-AB554. ${ }^{22}$

\section{Use of standardized optical diagnostic criteria}

To date, the NICE classification ${ }^{6,7}$ is the only validated criterion for NBI with and without magnification that describes realtime differentiation of nonneoplastic (type 1) and neoplastic (type 2) colorectal polyps, as well as lesions with deep submucosal invasion (type 3).

As mentioned above, it has been suggested that SSA/P features (clouded surface, indistinctive borders, irregular shape and dark spots inside crypts) should be integrated in the NICE classification. ${ }^{18}$ However, until such endoscopic and pathologic distinctions are further described, investigated and shown to be reproducible, it may be necessary to remove all lesions with SSA/P features and submit them to pathology.

\section{Use of standardized pathological diagnostic criteria}

It is important to use standardized diagnostic criteria for colorectal polyp histopathology. The World Health Organization criteria have been the most used. ${ }^{15}$ However, it must also be recognized that even the accuracy of standardized pathology is often less than $100 \%$. In clinical practice, many diminutive polyps are not retrieved following polypectomy, are unsuitable for analysis because of diathermy artifacts, or may be misclassified due to incorrect orientation or limited sectioning. Furthermore, the pathologists' rate of error in differentiating conventional ad- enomas from hyperplastic lesions in diminutive polyps may be as high as $10 \% .^{20}$ Besides, a well photodocumented polyp may sometimes reported by the pathologist as normal mucosa possibly because they section a part of the tissue surrounding the polyp rather than the lesion itself (Fig. 4). Moreover, interobserver concordance agreement among pathologists evaluating serrated lesions is at best moderate. ${ }^{17}$

\section{Use of appropriate technology}

Several studies in the literature have convincingly shown that white light alone is not sufficient for optical diagnosis, since the diagnostic accuracy achieved is significantly lower than with histopathology. ${ }^{23}$ The studies which have shown results comparable to histopathology have all used additional technology to white light-initially chromoendoscopy, and more recently narrow spectra technologies, among which NBI has been the most widely studied. High-magnification using CV260SL-Spectrum Lucera or CV-290-Lucera Elite videoprocessors and light source of Olympus have been shown to increase the proportion of high-confidence evaluations, ${ }^{8}$ but these systems are mainly available in Japan and the UK. Near focus view is an image enhancement tool available worldwide for CV-190-Exera III videoprocessors and light source developed by Olympus; it allows the colonoscope to get close to the polyp ( $2 \mathrm{~mm}$ ) and still remain in sharp focus (Fig. 5). The Veterans Affairs Colorectal Lesion Interpretation and Diagnosis (VALID) study has shown that this system also increases the number of lesions with highconfidence assessment. $^{9}$

The Olympus Pre-Freeze system, which stores the most recent image frames in a buffer in the videoprocessor and captures the sharpest still image, is also helpful. In addition, optimal processor settings for quality view (including enhancement A7 in white-light endoscopy and A8 in NBI, brightness and iris) should be obtained and a high-definition monitor should be in place at a suitable distance. 

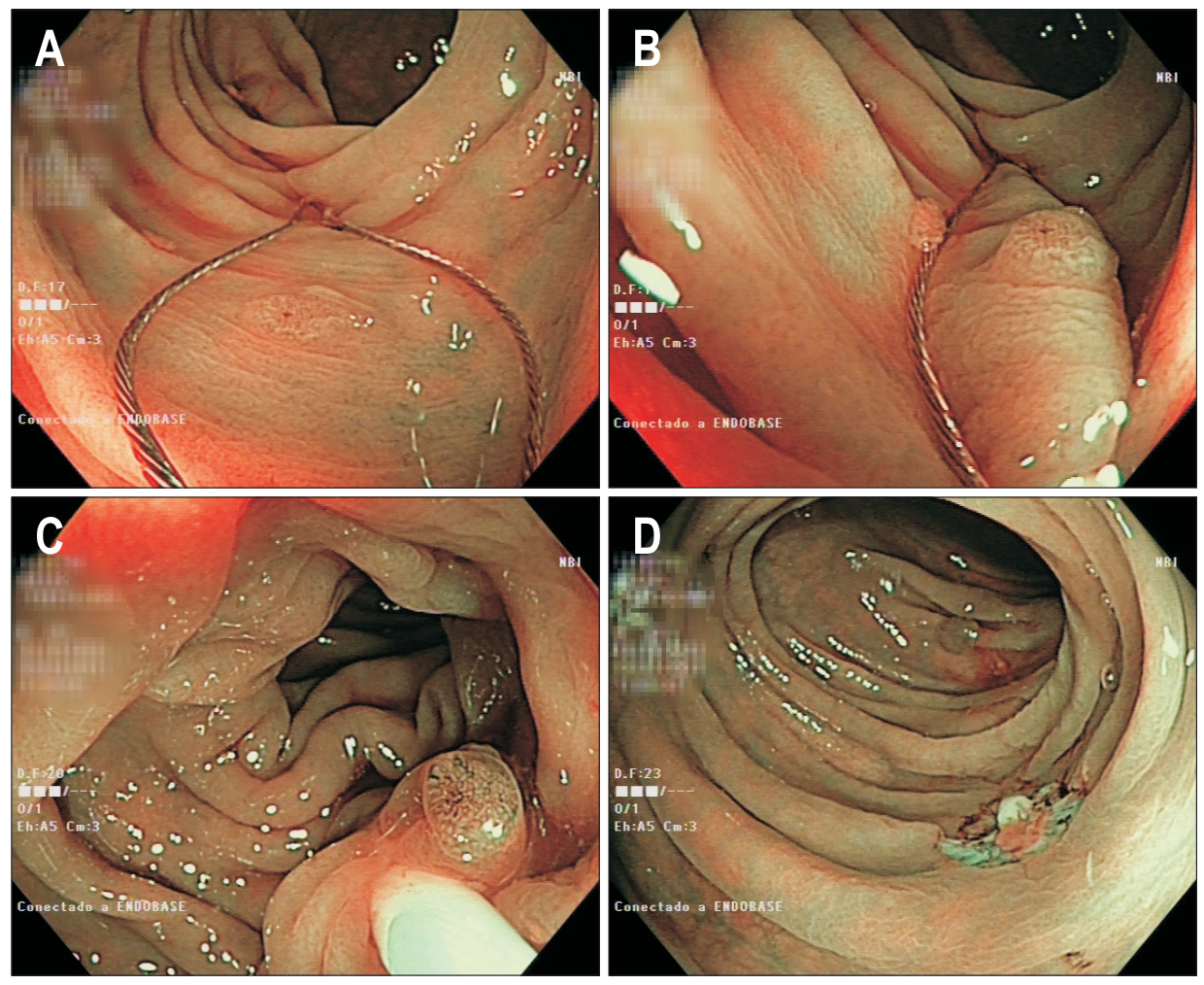

Fig. 4. (A-D) A 2-mm sessile (Is) adenoma was retrieved with a cold snare. A 5-mm specimen was referred for analysis, and histology showed normal mucosa.
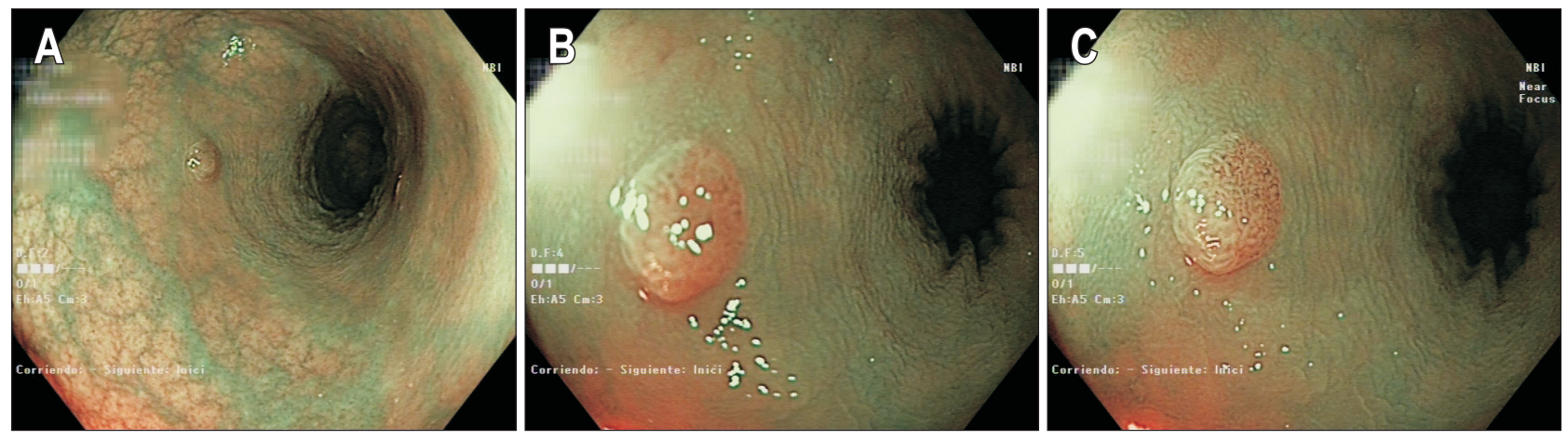

Fig. 5. Dual focus or magnification increases the number of high confidence lesions. (A) Excessively far view (capillary vessels cannot be clearly assessed). (B) Close-up view of the polyp without dual focus (capillary vessels and surface are not sharp). (C) Close-up view of the polyp with dual focus (capillary vessels and surface are sharp).

\section{Standardized training}

It has been suggested that four steps must be completed before a clinical skill can be put into practice: ${ }^{24}$ "knows" (in our case, the NICE classification); "knows how" (to image a polyp and to interpret its features); "shows" (to demonstrate real-time differentiation); "does" (to integrate optical diagnosis into clinical practice).

The PIVI statement highlights that "endoscopists who are expert in using this advanced imaging technology" are qualified to use optical diagnosis ${ }^{4}$ and the European Society of Gastrointestinal Endoscopy states that training is a prerequisite for the use of advanced imaging technology in clinical practice. ${ }^{25}$ Much like colonoscopy itself, knowledge of optical diagnosis is not innate and its use in colonoscopy reports should be avoided by endoscopists without previous training and demonstrated expertise.

There are a number of training modules available. ${ }^{26}$ Some studies have shown significant improvements in accuracy and in the proportion of high confidence predictions after a training module using high-definition photographs or videos of polyps. ${ }^{27-29}$ Although none of these studies used consecutively collected images or video content, their findings underscore the importance of learning before engagement in a formal study, or practice, of optical diagnosis. The formal training should be based on a validated tool, should be periodic, and should in- 
clude an in vivo component.

In addition, endoscopists should receive periodic feedback on their optical diagnosis performance. A recent study ${ }^{30}$ has shown that high NPV for the prediction of nonneoplasms with NBI was achieved and maintained in a group of endoscopists who participated in a standardized, continuous training program. Both NPV and surveillance interval agreement indicated high performance in the optical diagnosis of colorectal polyps and exceeded thresholds.

The implementation of optical diagnosis requires participants to recognize that learning is experiential: "a cyclic process of doing, noticing, questioning, reflecting, exploring concepts and models (evidence), and then doing again-only doing it better the next time (Plan-Do-Study-Act [PDSA] cycle). ${ }^{31}$ The iterative process of "checking" the correlation of endoscopic diagnosis to pathology findings is important. Without it, endoscopists miss a significant opportunity to continuously monitor and improve the quality of their optical diagnoses (Fig. 3). Optical diagnosis can only be implemented into clinical practice when endoscopists remain interested in learning, engaged, and committed to the process.

\section{Photo documentation}

The ability to take a clear image of the polyp to be diagnosed optically is of paramount importance. The first step is to optimize the processor, monitor, and capture settings. Second, optical diagnosis should not be attempted if images are deficient (Fig.
6). The image serves both as the record for the patient and as the audit for accreditation and quality assurance. Polyp imaging and optical diagnosis should be integrated into the endoscopy reporting system in order to ensure efficient real-time relay of the information and the reliability of the review. This archiving would allow both self and formal audits.

\section{IN SUMMARY}

Optical diagnosis of colorectal polyps can improve the costeffectiveness of screening colonoscopy. It avoids the cost of a pathology examination, improves efficiency related to the follow-up care, and reduces patients' anxiety, as they no longer have to wait for the pathology results.

Meta-analyses from centers with experience in optical diagnosis of colorectal polyps have shown results similar to those obtained with histopathology: an overall concordance of surveillance intervals of 93\%, and a negative predictive value for small polyps in the rectosigmoid colon of $\geq 90 \%$.

The implementation of optical diagnosis requires participants to recognize that learning is experiential: "A cyclic process of doing, noticing, questioning, reflecting, exploring concepts and models (evidence), and then doing again-only doing it better the next time (PDSA cycle)."

Confidence levels are important in the implementation of an optical diagnosis strategy. The use of confidence levels allows calibration and standardization between endoscopists with
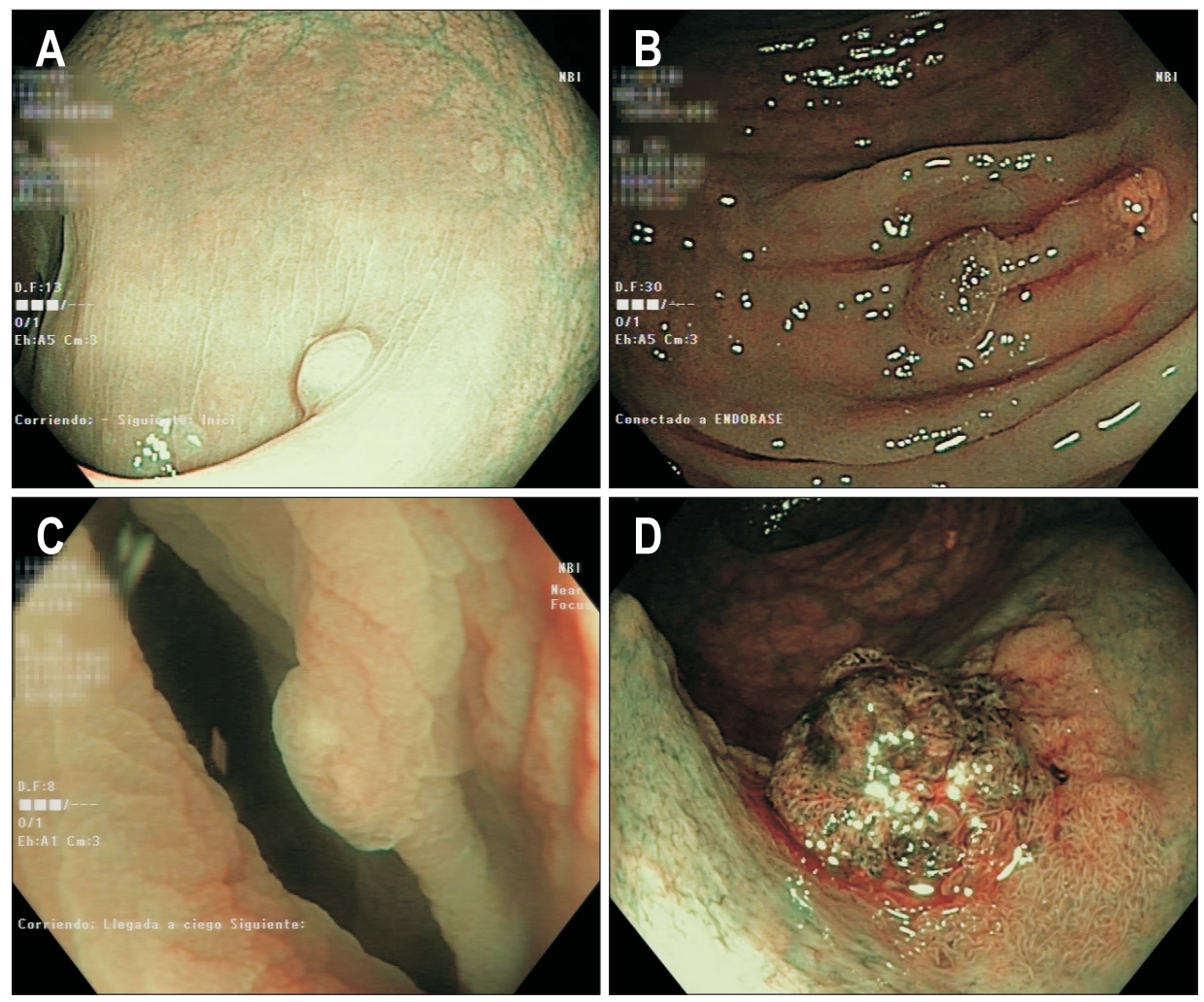

Fig. 6. Optical diagnosis should not be performed when image quality is low. (A) Too bright; (B) too dark; (C) lateral view (not frontal); (D) stool over the polyp. 
varying levels of diagnostic ability and reduces interobserver variation. Thus, if a polyp lacks clear endoscopic features that preclude a confident assignment of histology, the endoscopist should resect and submit it for pathologic assessment.

Photo documentation and archiving is a key component in both the study and the clinical implementation of optical diagnosis for accreditation and quality assurance.

\section{CONFLICTS OF INTEREST}

T.K. is a consultant for Olympus, America, and I.P. has no disclosures.

\section{REFERENCES}

1. Hassan C, Pickhardt PJ, Rex DK. A resect and discard strategy would improve cost-effectiveness of colorectal cancer screening. Clin Gastroenterol Hepatol 2010;8:865-869.

2. Ignjatovic A, East JE, Suzuki N, Vance M, Guenther T, Saunders BP. Optical diagnosis of small colorectal polyps at routine colonoscopy (Detect InSpect ChAracterise Resect and Discard; DISCARD trial): a prospective cohort study. Lancet Oncol 2009;10:11711178.

3. McGill SK, Evangelou E, Ioannidis JP, Soetikno RM, Kaltenbach T. Narrow band imaging to differentiate neoplastic and dkneoplastic colorectal polyps in real time: a meta-analysis of diagnostic operating characteristics. Gut 2013;62:1704-1713.

4. ASGE Technology Committee, Abu Dayyeh BK, Thosani N, et al. ASGE Technology Committee systematic review and meta-analysis assessing the ASGE PIVI thresholds for adopting real-time endoscopic assessment of the histology of diminutive colorectal polyps. Gastrointest Endosc 2015;81:502.e1-502.e16.

5. Rex DK, Kahi C, O’Brien M, et al. The American Society for Gastrointestinal Endoscopy PIVI (Preservation and Incorporation of Valuable Endoscopic Innovations) on real-time endoscopic assessment of the histology of diminutive colorectal polyps. Gastrointest Endosc 2011;73:419-422.

6. Hewett DG, Kaltenbach T, Sano Y, et al. Validation of a simple classification system for endoscopic diagnosis of small colorectal polyps using narrow-band imaging. Gastroenterology 2012;143:599-607.e1.

7. Hayashi N, Tanaka S, Hewett DG, et al. Endoscopic prediction of deep submucosal invasive carcinoma: validation of the NarrowBand Imaging International Colorectal Endoscopic (NICE) classification. Gastrointest Endosc 2013;78:625-632.

8. Iwatate M, Sano Y, Hattori S, et al. The addition of high magnifying endoscopy improves rates of high confidence optical diagnosis of colorectal polyps. Endosc Int Open 2015;3:E140-E145.

9. Kaltenbach T, Rastogi A, Rouse RV, et al. Real-time optical diagnosis for diminutive colorectal polyps using narrow-band imaging: the VALID randomised clinical trial. Gut 2015;64:1569-1577. 10. Puig I, López-Cerón M, Pellisé M, et al. Diagnostic accuracy of the nice classification for predicting deep submucosal invasion in colon lesions assessed in vivo preliminary results. Gastrointest Endosc 2016;83 (Suppl 5):AB404-AB405.

11. Kamiński MF, Hassan C, Bisschops R, et al. Advanced imaging for detection and differentiation of colorectal neoplasia: European Society of Gastrointestinal Endoscopy (ESGE) guideline. Endoscopy 2014;46:435-449.

12. Lieberman D, Moravec M, Holub J, Michaels L, Eisen G. Polyp size and advanced histology in patients undergoing colonoscopy screening: implications for CT colonography. Gastroenterology 2008;135:1100-1105.

13. Sano W, Sano Y, Iwatate M, et al. Prospective evaluation of the proportion of sessile serrated adenoma/polyps in endoscopically diagnosed colorectal polyps with hyperplastic features. Endosc Int Open 2015;3:E354-E358.

14. Snover DC. Update on the serrated pathway to colorectal carcinoma. Hum Pathol 2011;42:1-10.

15. Bosman FT, Carneiro F, Hruban RH, Theise ND. WHO classification of tumours of the digestive system. 4th ed. Lyon: World Health Organization, 2010.

16. Abdeljawad K, Vemulapalli KC, Kahi CJ, Cummings OW, Snover DC, Rex DK. Sessile serrated polyp prevalence determined by a colonoscopist with a high lesion detection rate and an experienced pathologist. Gastrointest Endosc 2015;81:517-524.

17. Wong NA, Hunt LP, Novelli MR, Shepherd NA, Warren BF. Observer agreement in the diagnosis of serrated polyps of the large bowel. Histopathology 2009;55:63-66.

18. IJspeert JE, Bastiaansen BA, van Leerdam ME, et al. Development and validation of the WASP classification system for optical diagnosis of adenomas, hyperplastic polyps and sessile serrated adenomas/polyps. Gut 2016;65:963-970.

19. Kaltenbach T, Rex DK, Wilson A, et al. Implementation of optical diagnosis for colorectal polyps: standardization of studies is needed. Clin Gastroenterol Hepatol 2015;13:6-10.e1.

20. Cross SS, Betmouni S, Burton JL, et al. What levels of agreement can be expected between histopathologists assigning cases to discrete nominal categories? A study of the diagnosis of hyperplastic and adenomatous colorectal polyps. Mod Pathol 2000;13:941944.

21. Rex DK. Narrow-band imaging without optical magnification for histologic analysis of colorectal polyps. Gastroenterology 2009;136:1174-1181.

22. Gupta N, Kaltenbach T, Sato T, et al. Diagnosis time determines the accuracy of optical diagnosis of diminutive polyp histology. Gastrointest Endosc 2013;77(5 Suppl):AB553-AB554.

23. Machida H, Sano Y, Hamamoto Y, et al. Narrow-band imaging in the diagnosis of colorectal mucosal lesions: a pilot study. Endoscopy 2004;36:1094-1098.

24. Miller GE. The assessment of clinical skills/competence/performance. Acad Med 1990;65(9 Suppl):S63-S67.

25. East JE, Vleugels JL, Roelandt P, et al. Advanced endoscopic imaging: European Society of Gastrointestinal Endoscopy (ESGE) 
Technology Review. Endoscopy 2016;48:1029-1045.

26. American Society of Gastrointestinal Endoscopy (ASGE). Optical diagnosis of colorectal polyps (OLV004) [Internet]. Downers Grove: ASGE; c2013 [cited 2017 Jul 24]. Available from: https:// asge.extendmed.com/index.php?option=com_asge\&view=learning module\&id=1309.

27. Raghavendra M, Hewett DG, Rex DK. Differentiating adenomas from hyperplastic colorectal polyps: narrow-band imaging can be learned in 20 minutes. Gastrointest Endosc 2010;72:572-576.

28. Rastogi A, Rao DS, Gupta N, et al. Impact of a computer-based teaching module on characterization of diminutive colon polyps by using narrow-band imaging by non-experts in academic and community practice: a video-based study. Gastrointest Endosc
2014;79:390-398.

29. Ignjatovic A, Thomas-Gibson S, East JE, et al. Development and validation of a training module on the use of narrow-band imaging in differentiation of small adenomas from hyperplastic colorectal polyps. Gastrointest Endosc 2011;73:128-133.

30. McGill SK, Soetikno R, Rastogi A, et al. Endoscopists can sustain high performance for the optical diagnosis of colorectal polyps following standardized and continued training. Endoscopy 2015;47:200-206.

31. Glasziou P, Ogrinc G, Goodman S. Can evidence-based medicine and clinical quality improvement learn from each other? BMJ Qual Saf 2011;20 Suppl 1:i13-i17. 